\title{
Application of the Maxillary Suture Obliteration Method For Estimating Age at Death in Greek Population
}

\author{
C. Apostolidou ${ }^{1 *}$, I. Eleminiadis ${ }^{1}$, T. Koletsa ${ }^{2}$, K. Natsis ${ }^{3}$, S. Dalampiras ${ }^{4}$, D. Psaroulis ${ }^{1}$, \\ S. Apostolidis ${ }^{3}$, A. Psifidis ${ }^{5}$, P. Tsikaras ${ }^{3}$ and S.N. Njau ${ }^{1}$ \\ 'Department of Forensic Medicine \& Toxicology, Medical School, Aristotle University of Thessaloniki, Greece \\ ${ }^{2}$ Pathology Department, Medical School, Aristotle University of Thessaloniki, Greece \\ ${ }^{3}$ Laboratory of Anatomy, Medical School, Aristotle University of Thessaloniki, Greece \\ ${ }^{4}$ School of Dentistry, Aristotle University of Thessaloniki, Greece \\ ${ }^{5}$ Department of Otorhinolaryngology, Medical School, Aristotle University of Thessaloniki, Greece
}

\begin{abstract}
A recently developed method for estimating adult skeletal age is based on maxillary suture obliteration. The purpose of this study is to test the accuracy of the revised maxillary suture method in estimating age at death on a skeletal sample from Greece. The sample consists of 271 skeletons (150 males, 121 females) of known age and sex and comes from several cemeteries of the country and from Human Skeletal Athens Collection. By using the method of Mann et al. we correctly estimated age for $89 \%$ of males and $84 \%$ of females. This finding suggests that the method is applicable to estimate the age-at-death in Greek population.
\end{abstract}

Keywords: Age estimation, maxillary suture, forensic science, human skeleton.

\section{INTRODUCTION}

The accurate determination of adult age at death is basic in the description of skeletal populations. Many macroscopic methods (qualitative and quantitative) and standards are often available for the adult age estimation from several skeletal indicators [1-9]. Most of them are based on morphological and degenerative changes in the human skeleton related to age. Until now none of these is wholly satisfactory to provide age estimates that are precise and accurate [10] because of the variability between different individuals and populations.

Forensic anthropology often utilizes multiple methods on the skeletal remains to create an age range for the identification of the skeleton. One of these age estimating techniques is the maxillary suture obliteration, introduced in 1987 by Mann and colleagues (referred to as original method) [11]. The original method was developed on a sample of 36 individuals, of known age and sex. The ancestry of this small sample was predominantly European. In 1991 Mann et al. published a revision of the maxillary suture method (referred to as revised method) [12]. The revised method was developed on a larger sample in an effort to overcome some of the restrictions of the original method. The sample included 186 individuals of European and African American ancestries.

Since then both methods (original [11] and revised [12]) have been subject to testing [13-15] and the results of these

*Address correspondence to this author at the Department of Forensic Medicine \& Toxicology, Medical School, Aristotle University of Thessaloniki, University Campus, 54124 Thessaloniki, Greece;

Tel: +302310999236; E-mail: katerinaapostolidoy@yahoo.com tests indicate that the obliteration of the maxillary sutures could be a useful age estimating indicator. But the question whether the revised maxillary suture method can be successfully applied to genetically different samples, still remains.

The purpose of this study is to investigate whether the revised maxillary suture method, developed on samples of American, European and African descent, can effectively estimate age at death in a sample from the Greek population.

\section{MATERIALS AND METHODOLOGY}

\section{Materials}

The sample consisted of 271 (150 males, 121 females) skeletons of known age and sex from cemeteries from several regions of Greece and Human Skeletal Athens Collection. The remains belonged to individuals who lived mainly in the second half of the $20^{\text {th }}$ century. The age distribution for the sample is shown in Table $\mathbf{1 .}$

In the sample were included only the maxillae that satisfied the criteria presented by Mann, who stated that the maxillary region must be complete and undamaged and the maxillae that have been sagitally sectioned should be excluded. None was rejected based on unusual morphological or pathological traits (such as extensive tooth loss, torus palatinous, edentulism)

\section{METHOD}

The maxillary suture method consists of dividing the maxilla into four sutures modified by Kopsch [16]: incisive (IN), anterior median palatine (AMP), transverse palatine (TP), and posterior median palatine (PMP). Although the rate and the amount of obliteration is quite variable, Mann 
Table 1. Sample Age Distribution

\begin{tabular}{|c|c|c|c|}
\hline Chronological Age (years) & Males & Females & 9 \\
\hline $20-24$ & 15 & 8 & 24 \\
\hline $25-29$ & 15 & 4 & 19 \\
\hline $30-34$ & 15 & 15 & 30 \\
\hline $35-39$ & 15 & 10 & 23 \\
\hline $40-44$ & 15 & 15 & 15 \\
\hline $45-49$ & 15 & 15 & 30 \\
\hline $50-54$ & 15 & 15 & 30 \\
\hline $55-59$ & 15 & 15 & 30 \\
\hline $60-64$ & 150 & 121 & 30 \\
\hline $64+$ & & & 271 \\
\hline All ages & 15 & & 30 \\
\hline
\end{tabular}

and colleagues found that the sutures follow a general pattern of obliteration with the IN being the first to obliterate and followed by the PMP, then the TP and finally the AMP. Obliteration was defined as any portion of a suture no longer visible. Then the percentage of obliteration for each suture calculating and assigning a value of obliteration between 0 and 4 (Table 2). The age estimate is based on the latest suture exhibits obliteration [11]. For example, if the IN is completely obliterated the next suture for examination is the PMP. If the PMP shows no obliteration but TP does, age estimation will be based on the TP suture.

Although the revised method was developed on the same principle as the original (skeletal age can be estimated by maxillary suture obliteration), there are some differences between the two. The revised method examines the greater palatine foramen and transverse palatine suture separately and thus expands the number of sutures and the number of age phases from four to five (Table 3 ). The revised method also suggests that an assessment of the hard palate (absence or present of teeth, condition of maxillary bone, alveolar resorption), should be considered in assigning the final age estimate [12]. Although that type of information can introduce a level of subjectivity in the method, however the condition of hard palate doesn't overcome the suture information.

Table 2. Evaluation of Obliteration

\begin{tabular}{|c|c|}
\hline Obliteration of Suture & Value of Obliteration \\
\hline \hline $0 \%$ & 0 \\
\hline $1-25 \%$ & 1 \\
\hline $26-50 \%$ & 2 \\
\hline $51-75 \%$ & 3 \\
\hline $76-100 \%$ & 4 \\
\hline
\end{tabular}

\section{RESULTS}

The revised maxillary suture method placed the $87 \%$ of our sample in the correct age category (Fig. 1). Age was correctly predicted more often for males than for females, like the results of other researches [11-15]. The proportion of correct age estimates was $89 \%$ in males and $84 \%$ in females (Table 4), but the sex difference was insignificant $\left(\mathrm{x}^{2}=1,510\right.$, $\mathrm{p}=0,219, \mathrm{df}=1)$.

The revised maxillary suture method yielded 35 incorrect age estimates of the total 271 individuals, most of them deviate from the actual age phase by \pm one phase (Table 5). In the current study where error exists, age was predominantly underestimated $(80 \%)$. Although the underestimates range from one to three phases, most of them differ from the actual age by one phase.

The precedence of correct age estimates for individuals over 50 years of age was $86 \%$ and for individuals under 50 years of age was $80 \%$. Of course, older individuals had a higher proportion of correct age estimates than younger individuals but also these differences are not statistically significant $\left[\chi^{2}=1,36, p=0,243, \mathrm{df}=1\right]$.

\section{DISCUSSION}

The estimation of adult skeletal age is one of the most difficult tasks in anthropology and forensic science because the condition of the human skeleton may be affected by many factors such as diet, sex, socioeconomic status, disease, environment and culture. Even in the same population two individuals may have different rate in skeletal metamorphosis and degeneration.

In 2005 Ginter published research of the effectiveness of the revised maxillary method [15]. The skeletons used in this study came from the Department of Anatomy and Cell Biology, University of Cape Town (UCT collection). The sample consisted of 155 individuals: 11 "black" (9 males, 2 females), 112 "white" (68 males, 44 females), 23 "colored" (13 males, 10 females) and 9 of unknown ancestry (6 males, 
Table 3. Age Range Phases for Original and Revised Method

\begin{tabular}{|c|c|c|c|}
\hline \multicolumn{2}{|c|}{ Original Method } & \multicolumn{2}{c|}{ Revised Method } \\
\hline Sutures & Age Range Phases & Sutures & Age Range Phases \\
\hline \hline IN & $<25$ & IN & $20-24$ \\
\hline PMP & $25-42$ & PMP & $30-34$ \\
\hline TP & $43-60$ & GPF & $35-50$ \\
\hline AMP & $60+$ & TP & $50+$ \\
\hline
\end{tabular}

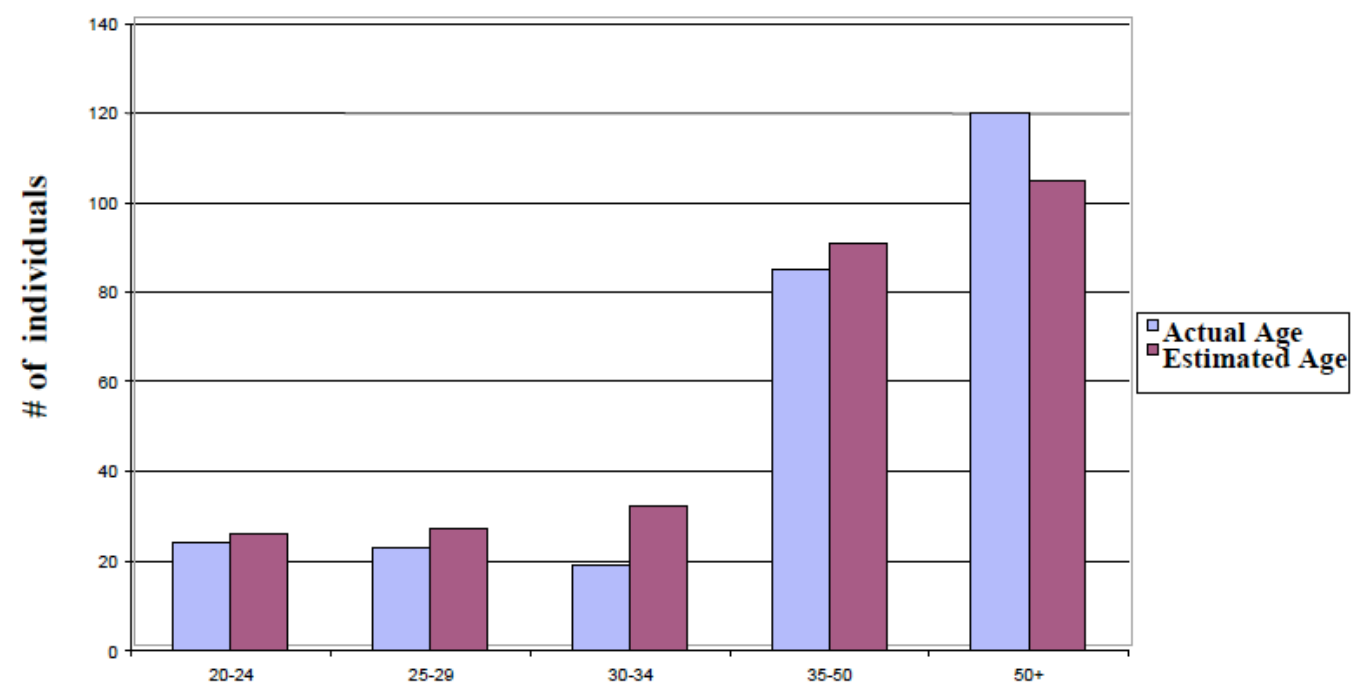

Age Phases

Fig. (1). The revised method: Comparison of the actual age distribution of the sample with the estimated age distribution.

Table 4. Revised Maxillary Suture Method: Numbers of Correct Age Phase Estimates by Five Years

\begin{tabular}{|c|c|c|c|c|}
\hline Five years & \multicolumn{2}{|c|}{ Males $(\mathrm{N}=150)$} & \multicolumn{2}{|c|}{ Females $(N=121)$} \\
\hline $25-29$ & 15 & 12 & 8 & 7 \\
\hline $30-34$ & 15 & 13 & 4 & 3 \\
\hline $40-44$ & 15 & 14 & 10 & 9 \\
\hline $45-49$ & 15 & 14 & 15 & 12 \\
\hline $50-54$ & 15 & 12 & 15 & 12 \\
\hline $65+15$ & 15 & 15 & 14 & \\
\hline Total & 150 & $134(89 \%)$ & 121 & $102(84 \%)$ \\
\hline
\end{tabular}

$\mathrm{N}^{1}$ Numbers of individuals in the sample whose actual ages fall within that age group. 
Table 5. The Number and Magnitude of Incorrect Age Phase Estimates Using the Revised Maxillary Suture Method

\begin{tabular}{|c|c|c|c|c|c|c|}
\hline & \multicolumn{3}{|c|}{ Males $(\mathrm{N}=150)$} & \multicolumn{3}{|c|}{ Females $(\mathrm{N}=121)$} \\
\hline \pm 1 & 13 & 4 & 9 & 11 & 2 & 9 \\
\hline \pm 2 & 3 & 1 & 2 & 7 & & 7 \\
\hline \multicolumn{7}{|l|}{ Total } \\
\hline incorrect & 16 & & & 19 & & \\
\hline Total $(+)$ & & 5 & & & 2 & \\
\hline Total (-) & & & 11 & & & 17 \\
\hline
\end{tabular}

3 females). By using the revised method, Ginter successfully aged $87 \%$ of those classified as having white ancestry. The proportion of correct age estimates for individuals of colored ancestry was $74 \%$ and for individuals of black ancestry was $73 \%$. Although the statistical difference wasn't significant between individuals of different backgrounds, the revised method was more accurate for the white ancestry.

In 2007 Sakaue and Adachi from Department of Anthropology of Tokyo, published a study in which they examined the revised maxillary suture method in Japanese [17]. They assessed the effectiveness of the method by using 375 (274 males and 101 females) Japanese skeletons of known age and sex. Although the proportion of correct age estimates was only $36,9 \%$ for males and $25,7 \%$ for females, the researchers found that the overestimates of age-at-death were seldom. This finding led the revised method to be widely accepted in Japan for estimating the minimum age-at-death in Japanese population.

In the present study we examined the revised maxillary suture method and estimated the effectiveness of the method in Greek population. The sample used in this research consisted of 271 (150 males and 121 females) Greek skeletons of known age and sex. The percentage of correctly aged individuals in this sample using the revised method was $87 \%$. At any given age, males exhibited more obliteration of the sutures than females, a finding consistent with the results of Mann et al. [12]. The correct age estimations were $89 \%$ for males and $84 \%$ for females. All sutures, in both sexes, start obliteration about at the same time, but in females the rate is slower. However, there is no statistically significant sex difference in the accuracy of age estimates.

Other researchers have noticed that cranial suture closure is influenced by population differences [18]. The rate of maxillary suture obliteration may also be affected by cultural or dietary differences. Nevertheless, the results of this study suggest that the revised maxillary suture method can be of value in estimating age at death in the Greek.

\section{CONCLUSION}

The revised maxillary suture method was applied to a large sample of skeletons from Greece with known age and sex, in an effort to test the performance of the method in a different ancestral sample. Age was correctly estimated for $89 \%$ of the males and $84 \%$ of the females without any statistically significant sex difference in the accuracy of the method. To sum up, the high proportion $(87 \%)$ of correct age estimation in the sample indicates that the method is applicable to estimate age at death in Greek samples.

\section{ACKNOWLEDGEMENTS}

We would like to thank Asterios Tsougas from the cemetery of Thermi Thessaloniki for taking time out of his work to help in the collection of the sample. We have to thank Dr. Sotiris Manolis and Dr. Kostas Iliopoulos from the Department of Animal and Human Physiology, University of Athens, Greece, for giving us access to the Human Skeletal Athens Collection. Many thanks also to Electric and Computer Engineer Anna Avdamalinou for the statistical analysis.

\section{REFERENCES}

[1] Lovejoy, C.O.; Meindl, R.; Pryzbeck, T.R.; Mensforth, R.P. Chronological metamorphosis of the auricular surface of the ilium : a new method for the determination of adult skeletal age at death. Am. J. Phys. Anthropol., 1985, 68, 15-28.

[2] Brooks, S.; Suchey, J.M. Skeletal age determination based on the pubis: a comparison of the Acsadi-Nemeskeri and Suckey-Brooks methods. Hum. Evol., 1990, 5, 227-238.

[3] Iscan, M.Y.; Loth, S.; Wright, R.K. Age estimation from the rib by phase analysis: white males. J. Forensic Sci., 1984, 29, 1094-1104.

[4] Iscan, M.Y.; Loth, S.; Wright, R.K. Age estimation from the rib by phase analysis: white females. J. Forensic Sci., 1984, 30, 853-863.

[5] Katz, D.; Suckey, J. Age determination of the male os pubis. Am. J. Phys. Anthropol., 1986, 69, 427-435.

[6] Nawrocki, S.P. Regression formulae for estimating age at death from cranial suture closure. In Forensic osteology: advances in the identification of human remains, Reichs KJ, Ed.; Wiley-Liss: New York, 1998; pp. 276-292.

[7] Buckberry, J.; Chamberlain, A.T. Age estimation from the auricular surface of the ilium: a revised method. Am. J. Phys. Anthropol., 2002, 119, 231-239.

[8] Osborn, D.L. Reconsidering the auricular surface as an indicator of age at death, Thesis, University of Western Michigan, 2000.

[9] Schmitt, A.; Murail, P.; Cunha, E.; Rouge, D. Variability of the pattern of aging on the human skeleton: evidence from bone indicators and implications on age at death estimating. J. Forensic Sci., 2002, 47, 1-7.

[10] Sokal, R.R.; Rohlf, F.J. Biometry, $3^{\text {rd }}$ Ed.; W.H. Freeman and Co: New York, 1995.

[11] Mann, R.W.; Symes, A.A.; Bass, W.M. Maxillary Suture Obliteration: aging the Human Skeleton Based on Intact or Fragmentary Maxilla. J. Forensic Sci., 1987, 32, 148-157. 
[12] Mann, R.W.; Jantz, R.L.; Bass, W.M.; Willey, P.S. Maxillary suture obliteration: A visual method for estimating skeletal age. $J$. Forensic Sci., 1991, 36, 781-791.

[13] Gruspier, K.L.; Mullen, G.J. Maxillary suture obliteration : A test of the Mann method. J. Forensic Sci., 1991, 36, 512-519.

[14] Ginter, J. Maxillary suture obliteration : A test of its effectiveness in predicting age at death. Abstract. Can. Soc. Forensic Sci. J., 2003, 36, 49-50.

[15] Ginter, J. A test of the effectiveness of the revised maxillary suture obliteration method in estimating adult age at death. J. Forensic Sci., 2005, 50,1303-1309.
[16] Kopsch, Fr. Nomina Anatomica, Knese K.-H., Ed.: Georg Thieme Verlag: Stuttgart, 1957, p. 128.

[17] Sakaue, K.; Adachi, N. Verification of the method for estimating age-at- death using maxillary suture obliteration in Japanese. Nihon Hoigaku Zasshi. 2007, 61, 121-128.

[18] Galera, V.; Ubelaker, D.H.; Hayek, L.C. Comparison of macroscopic cranial methods of age estimation applied to skeletons from the Terry Collection. J. Forensic Sci., 1998, 43, 933-9.

(C) Apostolidou et al.; Licensee Bentham Open.

This is an open access article licensed under the terms of the Creative Commons Attribution Non-Commercial License (http://creativecommons.org/licenses/by-nc/3.0/) which permits unrestricted, non-commercial use, distribution and reproduction in any medium, provided the work is properly cited. 